\title{
The Method of Inter-Transformation between Horizontal and Space Coordinate
}

\author{
Jianing ZHEN ${ }^{1,}$, , Guodong YANG ${ }^{1, b}$, Jingyu KANG $^{1, c}$, Xuqing ZHANG ${ }^{1, d}$ and \\ Heng WANG ${ }^{1, \mathrm{e}}$ \\ ${ }^{1}$ Jilin University \\ College of Geo-exploration Science and Technology \\ 938 West Democratic Avenue \\ Changchun 130026, Jilin Province, China \\ * Corresponding Author

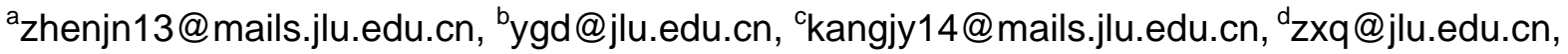 \\ ewangh15@mails.jlu.edu.cn
}

\begin{abstract}
Keywords: Coordinates transformation; Adjustment; Geodetic Height; Horizontal Coordinate; Abstract. Realizing coordinates transformation between WGS-84 datum and local datum is the key problems of GPS surveying.It is difficult to obtain the precise geodetic height in the national or local coordinate system which affects the accuracy of 7 parameter models. This paper puts forward a way of transformation which can achieve the WGS-84 to BJ-54 and WGS-84 to Xi'an 80 coordinate transformation combining with an "adjustment" method. It only needs to know the horizontal coordinate of each datum. The results of experiment show that in a certain longitude-latitude grid it is able to achieve the high accuracy horizontal coordinate transformation to meet the demand of the actual work in the case of lacking geodetic height.
\end{abstract}

\section{Introduction}

Usually the coordinates of GPS positioning is WGS-84 coordinates[1], and it should be transformed to national, local or engineering coordinates to meet the requirement of actual produced operation[2-3]. The methods of space coordinate transformation in the engineering commonly used bursa model[4-7], while the bursa model could be used only in spatial right-angle coordination system, as a consequence, we need to get geodetic height in the national or local coordinate system to transform the geodetic coordinates into spatial right-angle coordinates[8-12]. Although high precision WGS-84 coordinates are easier to obtain, it is difficult to get geodetic height in the national or local coordinate system by reason of its definition[13,14]. The overall precision of 7-parameter models is effected which would be used inconveniently.

There is little difference between national or local ellipsoid and WGS-84 ellipsoid in the process of coordinate transformation. Therefore, in a certain longitude-latitude grid, the shape of WGS-84 ellipsoidal surface is the same as regional ellipsoidal surface, and the main difference is the "relative position" of them, which includes the height difference, nonparallelism, and so on.

The coordinate transformation of surface can be understood as a problem of an "adjustment" of the two known surface. This paper presents the method of 7-parameter coordinate transformation based on "adjustment" method. It simulates, calculates and analysis the error characteristics and application scope of this method which needs not geodetic height and would be used conveniently.

\section{An “Adjustment” Method}

Take the WGS-84 to BJ-54 as an example, we assumed the ellipsoidal height $H$ of calculating points be zero in both WGS-84 and BJ-54 coordinate, which means that all of the calculation points were constrained on their ellipsoid surface, then, the seven parameters were solved with the method of the curved surface approximation. In this method, the ellipsoidal height has no practical significance, and 
was used as the standard to evaluating the approximation level of the two ellipsoids. This algorithm only adjusted the relative position of two ellipsoids but not change the curvature of the two ellipsoids. And the key of calculation is still the problem of 7-parameter coordinate transformation. Bursa model is in Eq. 1[15-17]:

$$
\left[\begin{array}{c}
\mathbf{X}_{b} \\
\mathbf{Y}_{b} \\
\mathbf{Z}_{b}
\end{array}\right]=\left[\begin{array}{c}
\Delta \mathbf{X} \\
\Delta \mathbf{Y} \\
\Delta \mathbf{Z}
\end{array}\right]+(1+k)\left[\begin{array}{l}
\mathbf{X}_{\boldsymbol{a}} \\
\mathbf{Y}_{\boldsymbol{a}} \\
\mathbf{Z}_{\boldsymbol{a}}
\end{array}\right]+\left[\begin{array}{ccc}
0 & \varepsilon_{z} & -\varepsilon_{y} \\
-\varepsilon_{z} & 0 & \varepsilon_{x} \\
\varepsilon_{y} & -\varepsilon_{x} & 0
\end{array}\right]\left[\begin{array}{c}
\mathbf{X}_{\boldsymbol{a}} \\
\mathbf{Y}_{\boldsymbol{a}} \\
\mathbf{Z}_{\boldsymbol{a}}
\end{array}\right]
$$

Where $\mathbf{X}_{\boldsymbol{a}}=\left(\mathrm{X}_{a}, \mathrm{Y}_{a}, \mathrm{Z}_{a}\right)^{T}$, which is considered to be the spatial right-angle coordinates in the original coordinate system, $\mathbf{X}_{\boldsymbol{b}}=\left(\mathrm{X}_{b}, \mathrm{Y}_{b}, \mathrm{Z}_{b}\right)^{T}$, which is considered to be the spatial right-angle coordinates in the objective coordinate system, $\Delta \mathrm{X}=(\Delta \mathrm{X}, \Delta \mathrm{Y}, \Delta \mathrm{Z})^{T}$, which is the translation parameters. $\varepsilon_{x}, \varepsilon_{y}, \varepsilon_{z}$ respectively represent the parameters of rotation matrix, $k$ is scale parameter.

The method of the "adjustment" was to make the coordinate in BJ-54 ellipsoid denoted by $\beta$ closing the corresponding coordinate in WGS-84 ellipsoid denoted by $\alpha$ in the study area, and the more visual demonstration was shown in Fig.1. We assumed the calculation points' ellipsoid height in both ellipsoid be zero. Actually, as shown in Fig.2. 3, the ellipsoid height of common points are different in the two coordinate system, and this difference value was calculated and used to measure the approximation level of the two ellipsoid surface during the iteration process, and this algorithm does not change the shape or the curvature of the ellipsoid surface, which means that the common points in two ellipsoid cannot totally overlap and there are errors in the common points, and these errors can be attributed as the model error. The model errors change by the different ellipsoid parameters, and can be calculated based on these ellipsoid parameters.

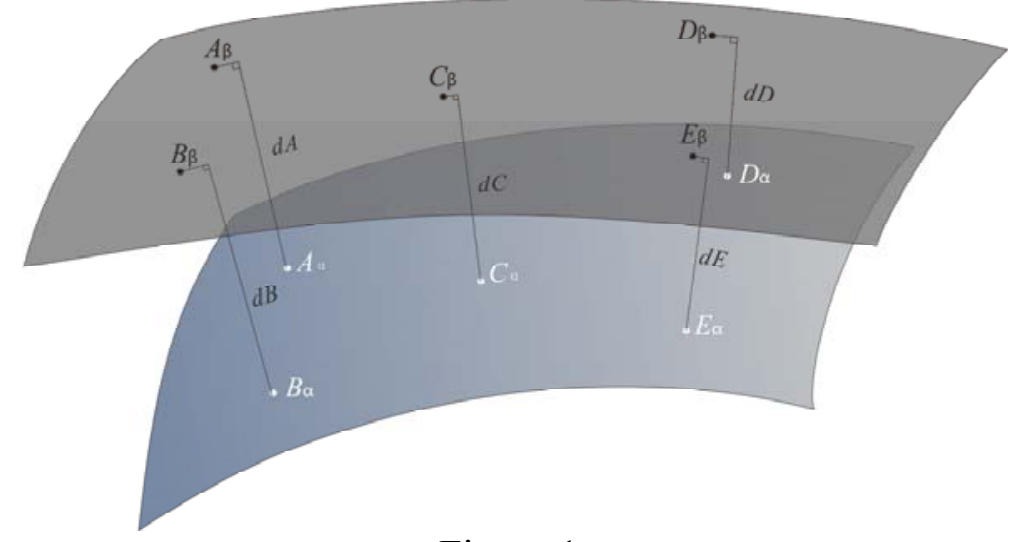

Figure 1.

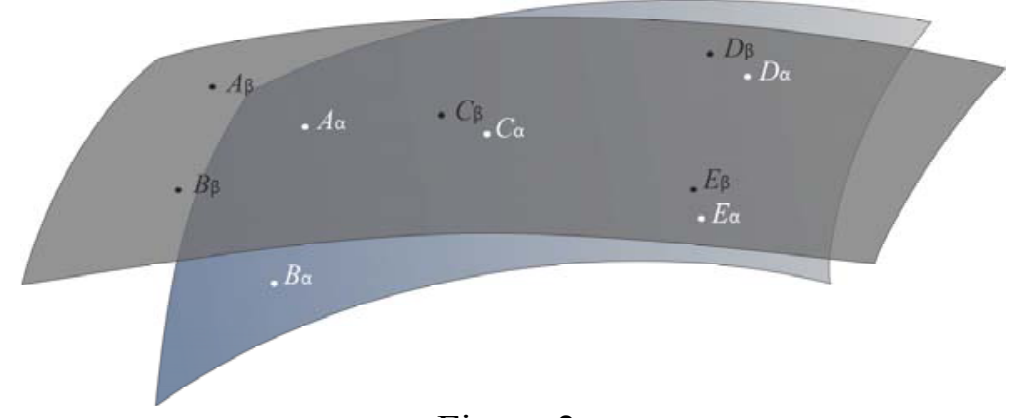

Figure 2.

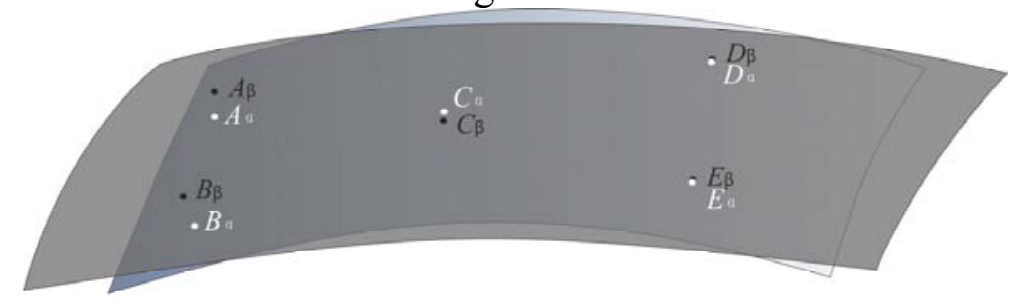

Figure 3.

And the flowchart of the iterative computations was shown in Fig. 4: 


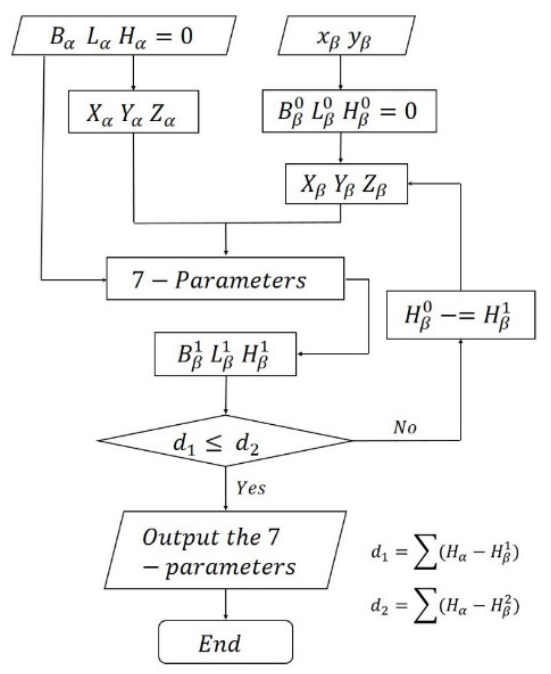

Figure 4.

Given the inconsistent of the curvature of two ellipsoids, the common points in two ellipsoid cannot overlap perfectly, as Fig.3 shown, which lead to the oscillation of ellipsoid height correction values in small range that can be calculated by the ellipsoid parameter. The horizontal errors caused by the oscillation of ellipsoid height was very small in middle and small scale of zone considering the error of triangular control points of BJ-54. The iteration stopped when $\mathrm{d} 1<\mathrm{d} 2$, that is, the stop condition of the iteration is the beginning of the oscillation. The approaching of the two ellipsoid surface in the calculating process can be attributed as that the minimization of common points on the two ellipsoid $\beta$ - $\alpha$ surface. When the iteration stopped, the two ellipsoid surface can be attributed as approaching by the distance minimization of common points and these distances approached average vertical distance

\section{Simulation Experiment}

In order to compare the applicability and stability of this method in different areas, select two areas to calculate, and choose two groups of different points in each area.

The calculation procedures are as follows:

At first: Suppose the 7 parameters which are from WGS-84 to BJ54 are: $\mathrm{dx}=10 \mathrm{dy}=$ $-20 \mathrm{dz}=15 \varepsilon_{\mathrm{x}}=5 \varepsilon_{\mathrm{y}}=2 \varepsilon_{\mathrm{z}}=1 \mathrm{~s}=20$. Where the basic unit of translation parameter is $\mathrm{m}$, the rotation parameter is second, the zooming parameter is ppm. According the value of B84、L84、 H84 of the points in each area to calculate the value of B54、L54、H54, then, the real value x54、 y54 of horizontal coordinate can be computed by Gauss projection the longitude of central meridian is $120^{\circ}$.

Secondly, supposing the values of the points' geodetic heights in WGS-84 coordinate system are zero, choose points to form coordinate groups to calculate its geodetic coordinate- B54', L54'. Compare the $\mathrm{x} 54$ ', y54' in the BJ-54 coordinate system which are calculated by Gauss projection with the real value of the horizontal coordinate $\mathrm{x} 54 、 \mathrm{y} 54$, and analysis the calculation results.

The difference between $\mathrm{x} 54, \mathrm{y} 54$ and $\mathrm{x} 54$ ', y54' of the four test data in each area is as shown in Table 1, where $\Delta \mathrm{x}=\mathrm{x} 54-\mathrm{x} 54^{\prime} \Delta \mathrm{y}=\mathrm{y} 54-\mathrm{y} 54$ '. 
Table 1

\begin{tabular}{llllll}
\hline & & & $117^{\circ}-123^{\circ} \mathrm{E}$ & & $117^{\circ}-123^{\circ} \mathrm{E}$ \\
& $26^{\circ}-34^{\circ} \mathrm{N}$ & & $48^{\circ}-56^{\circ} \mathrm{N}$ & \\
\hline group & point & $\Delta \mathrm{x}$ & $\Delta \mathrm{y}$ & $\Delta \mathrm{x}$ & $\Delta \mathrm{y}$ \\
\hline \multirow{4}{*}{1} & $\mathrm{~A}$ & 0.044 & -0.055 & 0.037 & 0.052 \\
& $\mathrm{~B}$ & 0.042 & 0.057 & 0.032 & 0.045 \\
& $\mathrm{C}$ & -0.016 & -0.043 & 0.012 & -0.038 \\
& $\mathrm{D}$ & 0.024 & 0.020 & -0.041 & -0.052 \\
& $\mathrm{E}$ & -0.039 & 0.052 & 0.025 & 0.055 \\
\hline \multirow{4}{*}{2} & $\mathrm{~F}$ & 0.021 & -0.018 & -0.013 & 0.021 \\
& $\mathrm{G}$ & -0.031 & 0.023 & 0.019 & 0.021 \\
& $\mathrm{H}$ & 0.013 & -0.007 & -0.039 & -0.050 \\
& $\mathrm{I}$ & 0.015 & 0.026 & -0.025 & 0.024 \\
& $\mathrm{~J}$ & -0.022 & 0.026 & 0.013 & -0.049 \\
\hline
\end{tabular}

Similarly, experiment on four groups data from WGS-84 to Xi'an-80 to obtain the difference between the real value and the calculated value of Xi' an- 80 horizontal coordinate. And the result was shown in Table 2:

Table 2

\begin{tabular}{llllll}
\hline & & $\begin{array}{l}117^{\circ} \sim 123^{\circ} \mathrm{E} \\
26^{\circ} \sim 34^{\circ} \mathrm{N}\end{array}$ & \multicolumn{1}{l}{$117^{\circ} \sim 123^{\circ} \mathrm{E}$} \\
$48^{\circ} \sim 56^{\circ} \mathrm{N}$ & \\
\hline group & point & $\Delta \mathrm{x}$ & $\Delta \mathrm{y}$ & $\Delta \mathrm{x}$ & $\Delta \mathrm{y}$ \\
\hline \multirow{4}{*}{1} & $\mathrm{~A}$ & 0.047 & -0.043 & 0.036 & -0.050 \\
& $\mathrm{~B}$ & -0.019 & 0.055 & -0.024 & 0.042 \\
& $\mathrm{C}$ & 0.025 & 0.010 & 0.022 & 0.005 \\
& $\mathrm{D}$ & -0.043 & 0.045 & -0.045 & 0.031 \\
& $\mathrm{E}$ & 0.014 & 0.057 & 0.016 & 0.055 \\
\hline \multirow{4}{*}{2} & $\mathrm{~F}$ & -0.035 & -0.047 & -0.023 & -0.020 \\
& $\mathrm{G}$ & 0.015 & -0.028 & 0.015 & 0.021 \\
& $\mathrm{H}$ & -0.003 & 0.030 & -0.019 & -0.000 \\
& $\mathrm{I}$ & -0.036 & -0.016 & 0.017 & 0.018 \\
& $\mathrm{~J}$ & 0.010 & 0.022 & 0.010 & 0.019 \\
\hline
\end{tabular}

The results show that the inner average precision of this model has high accuracy whose error is less than $6 \mathrm{~cm}$. The far from the center, the higher error is. Furthermore, error will decrease gradually with the increase of latitude and is associated with the curvature variation of meridian.

Taking the area as an example whose longitude ranges from $117^{\circ}$ to $123^{\circ}$ and latitude ranges from $26^{\circ}$ to $56^{\circ}$, use this method to carry out two groups of experiment. By the experiment data we can see all the maximum error is less than $5 \mathrm{~cm}$ and all the average error is less than $3 \mathrm{~cm}$. Different ways of choosing points will have different effect on coordinate transformation in this model. Furthermore, high accuracy horizontal coordinate can be obtained by this method in which longitude is the same and the latitude varies.

\section{Conclusions}

The results of study show that high accuracy horizontal coordinate transformation can be completed by using the idea of "adjustment" method in different areas .Because there is not accurate geodetic data in the astro-geodetic network of China, it avoids the effect of geodetic height error on coordinate transformation by using this method. As well as it is able to complete the high accuracy horizontal coordinate transformation to meet the demand of the actual work in the case of geodetic height unknown or inaccurate. 


\section{Reference}

[1] Wang Jiexian, Wang Jun, Lu Caiping: The Transformation Problems between the WGS-84 Coordinate and Beijing 54 Coordinate. J. Journal of Geodesy and Geodynamics. China, Vol. 23(2003), p. 70-73.

[2] Wang Jiexian, Qiu Yangyuan: Influence of Ellipsoidal Height Error on Seven-parameter Transformation. J. Journal of Geodesy and Geodynamics. China, Vol. 27(2007), p. 25-27.

[3] Xie Mingyu, Yao Yibin: A New Method for Solution of Seven-Parameter Transformation between 3D and 2D Spaces. J. Journal of Geodesy and Geodynamics. China, Vol. 28(2008), p. 104-109.

[4] Jiang Weiping, Ma Qiang, Liu Hongfei: The Method and Application of Coordinate Translation in CORS. J. Geomatics and Information Science of Wuhan University. China, Vol. 33(2008), p. 775-778.

[5] Wang Jiexian: Correlations among Parameters in 7-parameter Transformation Model. J. Journal of Geodesy and Geodynamics. China, Vol. 27(2007), p. 43-46.

[6] Kong Xiangyuan, Guo Jiming, Liu Zongquan: Fundamentals of Geodesy. (M. WuHan University Press, 2001).

[7] Yao Jili: Rigorous Formula for Direct Calculating Parameter in 3D Transformation. J. Bulletin of Surveying and Mapping. China, Vol. 5(2006), p. 7-10.

[8] Wang Zhijie, Wang Qing, Bi Feichao: Transformation Grid for Large-scale Positional Data from Geocentric Coordinate System to Ellipse-centered Coordinate System. J. Bulletin of Surveying and Mapping. China, Vol. 10(2013), p. 1-4.

[9] $\mathrm{Lu} \mathrm{Yu}$, Chen Yi, Zheng Bo: Applying Total Least Squares to Three-dimensional Datum Transformation. J. Journal of Geodesy and Geodynamics. China, Vol. 28(2008), p. 77-81.

[10] Shi Yimin: Contemporary Geodetic Control Survey. (M. SinoMaps press, 2003).

[11] He Lin, Liu Lintao, Xu Chaoqian: Study on Common Plane Coordinate System Conversion Plane Coordinate Conversion between BJ54, XA80 and CGCS2000. J. Bulletin of Surveying and Mapping. China, Vol. 9(2014), p. 6-11.

[12] Shen Yz, Chen Y, Zheng Dh: A Quaternion - Based Geodetic Datum Transformation Algorithm. J.Journal of Geodesy, Vol. 80(2006), p. 233-239.

[13] Papp. E: Geodetic datum transformation by quaternion. Publ Geomat, Vol .16(2013), p.17-28.

[14] Awange, J.L, Grafarend, E.W, Fukuda, Y: Exact solution of the nonlinear 7-parameter datum transformation by Groebner basis. Bul. di Geodesia e Scienze Affini, Vol. 63(2004), p. 117-127.

[15] Hooijberg: Practical Geodesy. M.Springer link: Berlin, Germany(2006), p. 17-59.

[16] Li, B.F, Shen, Y.Z, Li, W.X: The seamless model for three-dimensional datum transformation. M.Science China (Earth Science), Vol. 55(2012), p. 2099-2107.

[17] Za'voti J, Kalma'r: Several alternative possibilities for the solution of 3D non-linear similarity datum transformation compared to the Bursa-Wolf model. J.Publ Geomat, Vol. 17(2014), p.7-18. 